\title{
Philosophiques
}

\section{Fodor, la psychologie scientifique et les attributions de croyance}

\section{Pierre Jacob}

Volume 20, numéro 1, printemps 1993

URI : https://id.erudit.org/iderudit/027207ar

DOI : https://doi.org/10.7202/027207ar

Aller au sommaire du numéro

\section{Éditeur(s)}

Société de philosophie du Québec

\section{ISSN}

0316-2923 (imprimé)

1492-1391 (numérique)

Découvrir la revue

\section{Citer cet article}

Jacob, P. (1993). Fodor, la psychologie scientifique et les attributions de croyance. Philosophiques, 20(1), 131-158. https://doi.org/10.7202/027207ar
Résumé de l'article

La conception fodorienne de la psychologie scientifique soulève les trois problèmes suivants qui peuvent, je crois, être résolus. La condition de formalité est-elle compatible avec l'existence de lois psychologiques intentionnelles (qui font référence au contenu des états mentaux)? L'atomisme sémantique est-il compatible avec le fonctionnalisme? L'atomisme sémantique est-il compatible avec la systématicité des pensées humaines? Dans les trois premières sections du présent article, j'essaie de résorber ces tensions. Dans la quatrième et dernière section, j'examine la conception des attributions de croyance développée par Fodor dans "Substitution Arguments and the Individuation of Beliefs " selon laquelle la relation de croyance est une relation à quatre places entre une personne, un symbole (ou un véhicule), un rôle fonctionnel et un contenu propositionnel. Je fais valoir que Fodor est confronté à un dilemme : soit le rôle fonctionnel est un déterminant du contenu propositionnel, et Fodor doit rejeter l'atomisme sémantique; soit le rôle fonctionnel est un déterminant de l'efficacité causale d'un symbole, et il doit amender la condition de formalité.
Ce document est protégé par la loi sur le droit d'auteur. L’utilisation des services d'Érudit (y compris la reproduction) est assujettie à sa politique d'utilisation que vous pouvez consulter en ligne.

https://apropos.erudit.org/fr/usagers/politique-dutilisation/ 


\title{
FODOR, LA PSYCHOLOGIE SCIENIIFIOUE ET LES AITRIBUTIONS DE CROVANCE*
}

\author{
par \\ Pierre Jacob
}

RÉSUMÉ : La conception fodorienne de la psychologie scientifique soulève les trois problèmes suivants qui peuvent, je crois, être résolus. La condition de formalité estelle compatible avec l'existence de lois psychologiques intentionnelles (qui font référence au contenu des états mentaux) ? L'atomisme sémantique est-il compatible avec le fonctionnalisme? L'atomisme sémantique est-il compatible avec la systématicité des pensées humaines? Dans les trois premières sections $d u$ présent article, j'essaie de résorber ces tensions. Dans la quatrième et dernière section, j'examine la conception des attributions de croyance développée par Fodor dans "Substitution Arguments and the Individuation of Beliefs " selon laquelle la relation de croyance est une relation à quatre places entre une personne, un symbole (ou un véhicule), un rôle fonctionnel et un contenu propositionnel. Je fais valoir que Fodor est confronté à un dilemme: soit le rôle fonctionnel est un déterminant $d u$ contenu propositionnel, et Fodor doit rejeter l'atomisme sémantique; soit le rôle fonctionnel est un déterminant de l'efficacité causale d'un symbole, et il doit amender la condition de formalité.

ABSTRACT : Fodor's views about scientific psychology raise three puzzles: Is the Formality Condition compatible with there being intentional psychological laws (referring to the contents of mental states) ? Is Content Atomism consistent with Fonctionalism? Can Content Atomism be reconciled with the claim that human thoughts are

- Cet article est la version rédigée d'un exposé prononcé le 28 février 1992 au département de philosophie de l'UQAM. Je saisis cette occasion pour remercier les membres de ce département (notamment Robert Nadeau et Paul Dumouchel), les membres du département de philosophie de l'Université de Montréal (notamment François Lepage, Daniel Laurier, Michel Seymour) d'avoir organisé mon séjour parmi eux fin février-début mars 1992. Je remercie les auditeurs des trois exposés prononcés à cette occasion de leurs remarques et critiques. 
systematic? In the first three sections of this paper, I try and show how to solve the three puzzles. Finally, in the fourth section, I examine Fodor's recent view of beliefascriptions in "Substitution Arguments and the Individuation of Beliefs " according to which the belief relation is a four place relation between a believer, a symbol token (or vehicle), a functional role and a propositional content. I argue that Fodor faces a dilemma: either the functional role is a determinant of content, in which case Fodor must reject Content Atomism ; or it is a determinant of the causal efficacy of the vehicle, in which case he must amend his Formality Condition.

Mon but ici est de discuter la conception des attributions de croyance récemment proposée par Fodor dans un court article, «Substitution Arguments and the Individuation of Beliefs » ${ }^{\mathbf{I}}$. Cette théorie dépend d'une théorie de l'individuation des états de croyance et elle est présentée par Fodor dans le cadre d'une critique de l'argument frégéen contre les théories dénotationnelles du contenu. Comme le dit Fodor ${ }^{2}$, qui souscrit à la théorie dénotationnelle du contenu, «plus je vieillis, plus je suis enclin à penser que le contenu n'est autre que la dénotation ». Je supposerai, pour éviter les malentendus, que la théorie dénotationnelle s'applique aux expressions primitives qui ont une dénotation ou une référence. La doctrine suppose donc une distinction entre des contenus primitifs (indéfinissables) et des contenus dérivés par composition à partir des contenus primitifs. Fodor lui-même semble enclin à admettre une théorie du rôle fonctionnel (une théorie non dénotationnelle) pour des expressions dénuées de dénotation comme les symboles logiques (par exemple, les connecteurs propositionnels). La première chose à signaler sur la théorie dénotationnelle du contenu défendue par Fodor est qu'elle n'est pas sans évoquer la théorie dite de « la référence directe », selon laquelle la contribution d'un terme singulier à la proposition qu'il sert à exprimer n'est autre que son référent. Selon cette théorie, l'énoncé d'une phrase contenant un terme singulier exprime toujours une proposition singulière au sens de

I. Paru dans J.A. Fodor, A Theory of Content and Other Essays, Cambridge, Mass., MIT Press, 1991. Nous utiliserons l'abréviation SAIB lorsque nous nous référerons à cet article de Fodor.

2. $S A I B$, p. гб. 
David Kaplan ${ }^{3}$ - « toujours », c'est-à-dire dans tous les contextes, y compris les contextes d'attribution de croyance du type «François croit que Romain Gary était moustachu ».

Dans la section 4, je présenterai l'argument frégéen invoquant la non-substituabilité d'expressions coréférentielles dans certains contextes, argument dirigé contre la thèse dénotationnaliste, selon laquelle le contenu d'une expression référentielle n'est autre que sa dénotation. Je le comparerai à l'argument frégéen dirigé contre la théorie de la référence directe, selon laquelle la contribution propositionnelle d'un terme singulier est simplement d'introduire son référent dans la proposition « russelliemne singulière » (au sens de Kaplan) que l'énoncé de la phrase (qui contient le terme singulier) sert à exprimer. Je rappellerai brièvement la réponse d'un partisan de la théorie de la référence directe Nathan Salmon ${ }^{4}-$ au second argument frégéen. Je ferai ressortir ses faiblesses. Je ferai apparaître les avantages relatifs de la réponse fodorienne au premier argument frégéen. Finalement, je présenterai à Fodor un dilemme : soit il existe une tension entre sa théorie des attributions de croyance et la condition de formalité à laquelle doivent, selon la doctrine de « Methodological Solipsism $\gg^{5}$, obéir les processus mentaux ; soit il existe une tension entre sa théorie des attributions de croyance et sa répudiation du holisme du contenu ou son adhésion à une theorie purement dénotationnelle du contenu.

Pourquoi Fodor souscrit-il à la théorie dénotationnelle du contenu, lui qui souscrit à la théorie dualiste du contenu mental, c'est-à-dire à la distinction entre le contenu large et le contenu étroit? Pour répondre à cette question, et avant de présenter la théorie fodorienne des attributions de croyance et la réaction de Fodor à l'argument frégéen contre la théorie dénotationnelle recourant à la non-substituabilité d'expressions coréférentielles,

3. David Kaplan, « Dthat », dans P.A. French, T.E. Uehling $E$ H.K. Wettsein (dir.) Contemporary Perspectives in the Philosophy of Language, Minneapolis. University of Minnesota Press, I979.

4. Nathan Salmon, Frege's Puzzle, Cambridge, Mass., MIT Press, 1986.

5. J.A. Fodor, « Methodological Solipsism as a Resenrch Program in Cognitive Psychology », reproduit dans J.A. Fodor, Representations, Cambridge, Mass., MIT Press, 198I. 
je dois dire un mot de la perspective générale de Fodor sur la notion de contenu mental, dans la mesure où la motivation principale de sa réaction à l'argument frégéen est son adoption de la théorie dénotationnelle du contenu mental (dont j'ai déjà souligné la parenté avec la théorie de la référence directel. Or les raisons qu'a Fodor d'admettre la théorie dénotationnelle du contenu tiennent essentiellement à deux principes : son adhésion au projet de psychologie scientifique intentionnelle et son projet de naturalisation du contenu mental. Je ne parlerai ici que du premier, c'est-à-dire de la défense de la psychologie scientifique conjointe à la thèse de l'inéliminabilité du contenu mental dans la psychologie scientifique. On peut nommer son point de vue « le réalisme intentionnel »-j'y reviendrai dans la section 2. Ma stratégie consistera à présenter une résolution de trois énigmes auxquelles la conception fodorienne de la psychologie scientifique peut paraître à première vue s'exposer. La première énigme concerne la conciliation de la reconnaissance du contenu dans la psychologie scientifique avec la condition de formalité (section I). La deuxième énigme concerne la conciliation du fonctionnalisme avec la répudiation du holisme du contenu (section 2). La troisième énigme concerne la conciliation du rejet du holisme du contenu avec l'attribution de la systématicité aux pensées (section 3 ).

\section{Psychologie intentionnelle et condition de formalité}

Premièrement, Fodor maintient que les généralisations ceteris paribus de la psychologie cognitive scientifique mentionnent inévitablement le contenu des états mentaux intentionnels :

Toutes les généralisations concernant les états mentaux [...] s'appliquent aux attitudes propositionnelles en vertu du contenu des attitudes propositionnelles I...I les tentatives pour développer une psychologie cognitive sérieuse se fondent sur l'espoir que les généralisations de ce genre peuvent être systématisées et rendues rigoureuses [...] Or vous ne pouvez sauver ces génëralisations sans recourir à la notion de contenu d'un état mental ${ }^{6}$.

6. J.A. Fodor, Representations, Cambridge, Mass., MIT Press, I98I, p. 25-26; désormais Fodor, $R$. 
Un individu, Paul, forme, par exemple, la croyance que $q$ parce qu'il a la croyance que $p$ et la croyance conditionnelle que si palors q. Comme le souligne Fodor $^{7}$, les relations sémantiques entre les contenus des attitudes sont parallèles aux relations causales entre les attitudes ou les états de croyance. La proposition que $q$ est la conclusion d'une inférence correcte dont les prémisses sont les propositions $p$ et si palors $q$, et dont la règle d'inférence est le modus ponens. Parallèlement, le sens commun et la psychologie scientifique (selon Fodor) tiennent la croyance que $p$ et la croyance que si $p$ alors $q$ pour des causes de la croyance que $q$, si tant est que Paul conclut effectivement $q$ des deux prémisses ${ }^{8}$. Les généralisations intentionnelles de ce genre s'appliquent à n'importe quel contenu en général - et non à un contenu spécifique. Elles supposent simplement que soient préservées les relations d'identité et de différence entre les contenus exprimés par les $\langle p »$ et les $\langle q\rangle$. Elles présupposent donc que les contenus obéissent à des conditions d'individuation.

I...I le rôle causal d'une attitude reflète le rôle sémantique de la proposition qui est son objet l... l'une des contraintes sur l'assignation de contenus propositionnels aux états mentaux est que ceux-ci doivent ètre régis par un système de relations causales appropriées aux relations sémantiques entre ceux-là l...I nous pouvons assigner de manière non arbitraire des propositions comme objets des attitudes propositionnelles parce qu'il y a un isomorphisme entre d'une part le réseauu engendré par les relations sémantiques constitutives entre propositions et d'autre part le réseau engendre par les relations causales constitutives entre les états mentaux ${ }^{9}$.

Le but de la psychologie scientifique est d'expliquer ce mystérieux parallélisme des relations sémantiques entre les contenus des états mentaux intentionnels et des relations causales entre les états mentaux.

Comme chacun le sait, dans le projet de psychologie scientifique esquissé par Fodor depuis The Language of Thought et baptisé « théorie représentationmelle de l'esprit », le contenu

7. Chapitres I et 3 de J.A. Fodor, Psychosemantics, Cambridge, Mass., MIT Press, I987; desormais Fodor, $P$.

8. Cf. Ibid., chap. $\mathbf{I}^{-3}$.

9. Ibid., p. $7^{8}-79$. 
d'une attitude est réduit au contenu d'une formule du langage de la pensée:

Pour tout organisme $O$ et toute attitude $A$ vis-à-vis de la proposition $P$, il existe une relation (« computationnelle / fonctionnelle $\gg) R$ et une représentation mentale $M$ telle que

$M$ signifie que $P$

et

$O$ a $A$ ssi $O$ est unie à $M$ par $R^{\text {to }}$.

Par l'hypothèse du langage de la pensée, les formules du langage de la pensée ont, comme les symboles des langues naturelles publiques, des propriétés syntaxiques et des propriétés sémantiques. La psychologie cognitive scientifique est tenue par Fodor pour une théorie des processus de modification des symboles du langage de la pensée. L'une des questions est de savoir la part respective que jouent les propriétés syntaxiques et les propriétés sémantiques des symboles dans cette conception de la psychologie scientifique.

Fodor souscrit deuxièmement à la condition de formalité, selon laquelle, en substance, les processus mentaux pertinents pour la psychologie cognitive scientifique ne peuvent détecter que la forme ou les propriétés syntaxiques, non le contenu ou les propriétés sémantiques, des symboles mentaux. Grâce à la condition de formalité, les symboles mentaux sont supposés entrés dans des interactions causales les uns avec les autres en vertu de leurs propriétés syntaxiques (non sémantiques). La condition de formalité est supposée résoudre le mystère de l'efficacité causale des propriétés sémantiques des symboles - comment le contenu d'un symbole peut-il avoir une efficacité causale? -, qui n'est autre que le mystère du parallélisme des relations de contenus des états mentaux et des relations causales entre les états :

L'idée est de combiner l'hypothèse des représentations mentales avec la métaphore de l'ordinateur. Les ordinateurs nous montrent comment relier les propriétés sémantiques des symboles à leurs propriétés causales I...I Vous reliez les propriétés causales d'un symbole à ses propriètés sèmantiques via sa syntaxc. Il est facile l...) d'imaginer des symboles concrets particuliers (tokens) interagissant causalement en vertu de leurs structures syntaxiques [...] Les ordinateurs sont une solution au problème de la médiation entre les propriétés 
causales des symboles et leurs propriétés sémantiques. Si. donc, l'esprit est une sorte d'ordinateur, on voit comment une théorie des processus mentaux peut réussir $\mid . . . l$ à expliquer comment il peut exister des relations non arbitraires de contenu entre des pensées causalement reliées les unes aux autres ${ }^{\text {II }}$.

Autrement dit, les propriétés sémantiques des symboles mentaux semblent n'avoir d'efficacité causale que par proxy, via l'efficacité causale de leurs propriétés syntaxiques.

Stich $^{12}$ a reproché à Fodor de vouloir le beurre et l'argent du beurre en maintenant que la psychologie scientifique doit être intentionnelle (ou mentionner le contenu des attitudes propositionnelles) et en souscrivant conjointement à la condition de formalité :

Comment Fodor peut-il à la fois recommander que les généralisations cognitives s'appliquent aux états mentaux en vertu de leurs contenus et que « seules les propriétés non sémantiques des représentations mentales peuvent servir à déterminer quelles operations s'y appliquent?

Cette première énigme cesse d'en être une dès lors qu'on distingue, selon les nombreuses recommandations explicites de Fodor, entre les généralisations intentionnelles de la psychologie et les processus mentaux ou les mécanismes d'implémentation des généralisations. Dans la conception fodorienne ${ }^{\mathrm{I} 3}$, les généralisations intentionnelles de la psychologie sont supposées être implémentées par les processus mentaux, au sens où les lois de Mendel sont supposées implémentées par les mécanismes biochimiques du code génétique:

Nombreux sont les cas dans les sciences respectables où une loi relie une paire de propriétés sans que les propriétés en question ne figurent dans les mécanismes d'implémentation de la loi. C'est, par exemple, une loi approximative que les parents de haute taille ont des enfants de haute taille. Et il existe des mécanismes d'implémentation de cette loi. Mais la propriété d'être de haute taille, à la différence des propriétés génétiques, ne figure pas dans les mécanismes d'implémentation ${ }^{14}$.

\footnotetext{
II. Fodor, $P$, p. r8.

12. S. Stich, From Folk Psychology to Cognitive Science, Cambridge, Mass., MIT Press, 1983, p. 188 .

13. Fodor, $P$, p. 139-140 et « Making Mind Matter More».

14. Ibid., p. 140 .
} 
Autrement dit, selon le modèle de la causalité qui prévaut dans l'explication causale génétique de la transmission de la propriété être de haute taille par les parents à leur progéniture, une propriété génétique d'un parent a une efficacité causale dans deux processus causaux indépendants : d'une part elle joue un rôle dans l'acquisition ontogénétique par le parent de la propriété phénotypique d'être de haute taille; d'autre part elle joue un rôle dans l'acquisition par sa progéniture d'un génotype susceptible à son tour de jouer un rôle dans l'acquisition ontogénétique par l'enfant de la propriété d'être de haute taille. Dans aucun de ces deux processus la propriété du parent d'être de haute taille ne joue un rôle causal. Semblablement, si on admet que les lois intentionnelles régissant les symboles mentaux (et mentionnant leurs propriétés sémantiques) sont implémentées par des processus mentaux qui ne détectent que la forme syntaxique des symboles mentaux, on conclura que le contenu lou les propriétés sémantiques) des symboles mentaux ne joue pas, contrairement aux propriétés syntaxiques des symboles, de rôle causal.

D'une manière générale, c'est un trait de toutes les sciences spéciales, selon Fodor, que les généralisations appartenant à une discipline de niveau $n$ sont implémentées par des mécanismes révélés par une discipline de niveau $n-I^{15}$. Je pense que cette stratégie générale permet de dénouer la tension notée par Stich ${ }^{16}$.

\section{Répudiation du holisme du contenu, psychofonctionnalisme et fonctionnalisme sémantique}

Le trait le plus singulier de la position prise par Fodor depuis Psychosemantics dans la philosophie contemporaine de l'esprit est sa répudiation du holisme du contenu. Pour définir ce que Fodor baptise holisme du contenu, il faut définir ce qu'il appelle une

15. Cf. « Making Mind Matter More ».

16. Dans « Are Mental Properties Causally Efficacious?», Grazer Philosophische Studien. $39,51^{-7} 73$, j'ai exprimé mes doutes sur l'efficacité causale que Fodor est enclin à accorder aux propriétés syntaxiques des symboles mentaux et dont il prive les propriétés sémantiques. Je crois que Fodor joue sur l'ambiguïté du mot « forme » entre le contour (shape) d'un symbole et ses proprictés syntaxiques lorsqu'il ecrit que "la syntaxe d'un symbole particulier concret peut detenniner les causes et les effets de son instanciation de la même manière que la géométrie d'une clef détermine les semures quielle ouvrira ». 
«liaison épistémique »: une proposition $q$ est une liaison épistémique de la proposition $p$, pour un agent $A$, si $A$ tient la vérité de $q$ pour pertinente à l'évaluation de $p$. « Le holisme du contenu est l'idée que l'identité - spécifiquement, le contenu intentionnel - d'une attitude propositionnelle est déterminée par la totalité de ses liaisons épistémiques. ${ }^{17} \gg$

Pourquoi Fodor répudie-t-il si vigoureusement le holisme du contenu ? La réponse est qu'il y a un conflit entre le holisme du contenu et les exigences de la psychologie scientifique intentionnelle - laquelle « parvient à généraliser en quantifiant sur tous les organismes qui sont dans un état intentionnel spécifique $\gg^{8}$. Selon le projet de psychologie scientifique intentionnelle, tous les individus qui peuvent raisonnablement être crédités du désir que $q$ et de la croyance que non $q$ faute de $p$ devraient pouvoir être crédités ceteris paribus de l'intention d'agir de sorte à réaliser l'état de choses susceptible de rendre $p$ vraie. Mais, comme l'écrit Fodor :

\begin{abstract}
[...] si - comme c'est sûrement le cas - les gens diffèrent dans leurs estimations de pertinence épistémique, et si nous suivons le holisme du contenu en individualisant les états intentionnels par la totalité de leurs liaisons épistémiques, jamais deux personnes (ni même deux tranches temporelles d'une même personne) ne seront dans le même état intentionnel. Deux personnes ne seront jamais subsumées par les mêmes généralisations intentionnelles. Donc, les généralisations intentionnelles ne réussiront jamais à généraliser. Donc, il n'y a pas d'espoir pour la psychologie intentionnelle ${ }^{19}$.
\end{abstract}

Pour sauver la psychologie intentionnelle, Fodor rejette donc le holisme du contenu.

Mais la répudiation du holisme du contenu est-elle compatible avec le maintien du fonctionnalisme? Appelons «psychofonctionnaliste » la réponse fonctionnaliste à la question ontologique du rapport entre les états mentaux intentionnels d'un individu et les états cérébraux de l'individu. Dans la conception psychofonctionnaliste des états mentaux intentionnels - ou du rapport entre les états mentaux intentionnels et les états cérébraux - d'un individu, les états mentaux concrets particuliers (tokens) sont tenus pour des états cérébraux concrets 
particuliers de l'individu définis par leurs relations causales avec les inputs (ou entrées sensorielles), les autres états et les outputs (ou sorties comportementales). Étant donné le parallélisme déjà mentionné entre d'une part les relations sémantiques entre les contenus des états mentaux et d'autre part les relations causales entre les états mentaux, il n'y a qu'un pas entre la notion de rôle causal d'un état et la notion de rôle inférentiel (ou conceptuel). Il n'y a qu'un pas entre la conception psychofonctionnaliste, selon laquelle les états mentaux particuliers sont des états cérébraux particuliers caractérisés par leurs relations causales avec les inputs, les autres états et les outputs, et l'idée que les états mentaux sont des états cérébraux caractérisés par leur rôle inférentiel ou conceptuel - par le rôle qu'ils jouent dans les inférences que l'individu dont ils sont les états est enclin à accomplir. Si (comme Fodor le proclame), vous rejetez la distinction analytique / synthétique et si vous acceptez la conception psychofonctionnaliste des états mentaux, alors

I...I vous ne pouvez avoir de distinction de principe entre les relations causales entre états mentaux qui déterminent le contenu (c'est-à-dire l'identité) d'un état et les relations causales qui ne le déterminent pas. La conséquence immédiate est que vous ne pouvez pas avoir de fonctionnalisme sans le holisme; si n'importe quelle fonction d'un état mental peut affecter son contenu, alors toutes ses fonctions peuvent affecter le contenu. Mais si toutes les fonctions peuvent affecter le contenu, alors les membres d'une paire d'états mentaux particuliers n'auront jamais le même contenu et il $n^{\prime} y$ aura pas d'explication psychologique par subsomption sous une loi intentionnelle ${ }^{20}$.

En un mot, le rejet de la distinction analytique / synthétique combiné au fonctionnalisme semble impliquer le holisme du contenu. Or le holisme du contenu est, nous l'avons vu, incompatible avec la psychologie scientifique intentionnelle. La deuxième énigme dont je veux parler est donc de savoir comment on peut répudier le holisme du contenu et conserver le fonctionnalisme - ce que prétend manifestement faire Fodor -, tout en rejetant la distinction analytique / synthétique. Pour faire état de la

20. J.A. Fodor, A Theory of Content and Other Essays, Cambridge, Mass., MIT Press, I99I ; voir Introduction, $\mathrm{p}$. $x$. 
continuité de la pensée de Fodor sur ce point, je citerai un passage de l'Introduction de Fodor à Representations (de ig8I) :

l... l'intuition préthéorique dit que les croyances sont des causes, et de surcroit que ce sont des causes distinctes des stimuli, des réponses et des dispositions. Le fonctionnalisme souscrit à tout cela. Mais l'intuition préthéorique dit aussi que les croyances sont des causes qui ont un contenu, et sur ce point, le fonctionnalisme est neutre l...] Le fonctionnalisme est notamment compatible avec le fait que les processus de comportement ont des causes mentales, mais qu'il n'existe pas d'explication cohérente de l'idée qu'une croyance a l'effet qu'elle a en vertu du fait qu'elle est la croyance que $\mathrm{P}[. . . . \mathrm{l}$ si le fonctionnalisme est vrai et instructif, ce n'est pas une solution au problème qui rend la psychologie cognitive spéciale parmi les sciences - à savoir le recours (apparemment inéliminable) à des étiologies dans lesquelles les objets qui ont un contenu propositionnel figurent comme agents causaux. Sur ce point, le fonctionnalisme est neutre et donc non informatif ${ }^{2 I}$.

Étant donné que le fonctionnalisme apparaît comme une prémisse dans le raisonnement aboutissant au holisme du contenu, et étant donné que Fodor rejette le holisme du contenu qu'il juge incompatible avec la psychologie scientifique intentionnelle, comment Fodor peut-il maintenir le fonctionnalisme? La réponse à cette énigme est que Fodor distingue entre le psychofonctionnalisme auquel il souscrit et le fonctionnalisme sémantique qu'il rejette sauf sans doute pour le contenu exprimé par des expressions comme les connecteurs propositionnels. Le psychofonctionnalisme est la conception du rapport ontologique entre les états mentaux d'un individu et ses états cérébraux. Selon le fonctionnalisme sémantique, le contenu d'un état mental intentionnel n'est autre que le rôle causal ou le rôle inférentiel de l'état mental ou son potentiel d'interaction avec d'autres états mentaux intentionnels. Autrement dit, Fodor pense que le fonctionnalisme sémantique - non le psychofonctionnalisme - sert de prémisse dans le raisonnement qui aboutit au holisme du contenu et le rejet du holisme du contenu n'implique, selon lui, que le rejet du fonctionnalisme sémantique. Voici les termes dans lesquels Fodor exprime explicitement son adhésion à la conception psychofonctionnaliste des états (mentaux intentionnels) et sa répudiation concomitante du fonctionnalisme sémantique: 
1... être une croyance, c'est avoir les relations appropriées aux entrées, aux sorties et aux autres états mentaux l...l lce qui n'implique pas quel être la croyance que p, être une croyance avec un certain contenu, ce soit avoir les relations appropriées aux entrées, aux sorties et aux autres états mentaux l...l une définition fonctionnaliste de «croire» accomplirait la tâche fixée au psychofonctionnalisme en philosophie de l'esprit, mème si nous n'adoptons pas une conception fonctionnaliste des contenus de croyances, une conception fonctionnelle de ce que c'est que de croire que $p^{22}$.

l... le rôle fonctionnel n'exerce, selon moi, approximativement aucune contrainte sur le contenu d'un état mental; il exerce des contraintes sur son caractere. Selon moi, croire, contrairement à croire que $p$, est fonctionnellement dëfini ${ }^{23}$.

Imaginons que Jean espère qu'il neigera dimanche (parce qu'il a l'intention d'aller faire du ski dimanche). La combinaison de son espoir et de sa croyance qu'il neigera dimanche engendre sa joie. Jeanne redoute qu'il neige dimanche (parce qu'elle a l'intention de planter des pétunias). La combinaison de sa crainte et de sa croyance qu'il neigera engendre son humeur chagrine. Selon le sens commun, l'espoir de Jean et la crainte de Jeanne ont le même contenu. Mais à l'évidence ils ont des rôles causaux distincts puisqu'en interagissant avec une croyance ayant un seul et même contenu, ils engendrent des états distincts. Fodor en tire argument pour souligner le fossé entre le psychofonctionnalisme qu'il accepte et le fonctionnalisme sémantique qu'il rejette. Cette stratégie a nombre de conséquences.

Au début du chapitre IV de Psychosemantics ${ }^{24}$, consacré au problème de la naturalisation du contenu, Fodor se demande « en quoi consiste le fait pour un système physique d'avoir des états intentionnels $\gg$. Et il distingue mystérieusement cette question de la question de savoir en quoi consiste le fait pour un système physique d'être un «système intentionnel $»^{25}$. En quoi peut donc consister la différence entre le fait d'avoir des états intentionnels et le fait d'être un système intentionnel ? Par hypothèse, les systèmes intentionnels sont supposés avoir des attitudes propositionnelles. Or être une attitude propositionnelle (une croyance,

\footnotetext{
22. Fodor, P., p. 69-70.

23. Ibid., p. 88.

24. Ibid., P. 97.

25. Ibid., p. I63.
} 
par exemple), c'est, pour un état cérébral - conformément au psychofonctionnalisme -, entrer dans des relations causales appropriées avec des entrées, des sorties et d'autres attitudes propositionnelles. En revanche, avoir des états intentionnels, c'est, pour un système physique, avoir des états représentationnels - c'est-à-dire des états informationnels d'un type particulier (des états informationnels qui obéissent, selon Fodor, à la condition de dépendance causale asymétrique ${ }^{26}$ ). Un organisme ou un artefact peuvent donc avoir des états intentionnels ou représentationnels sans avoir des attitudes propositionnelles. Pour avoir des états intentionnels, un organisme doit avoir des états informationnels obéissant à la condition de dépendance causale asymétrique.

Fodor distingue donc trois choses : la notion d'information (qu'il réduit à la notion de dépendance nomique entre propriétés); la notion de contenu intentionnel (qui est une espèce d'information restreinte puisqu'elle se réduit à une dépendance asymétrique entre des relations nomiques entre propriétés) ; enfin, la notion d'attitude propositionnelle qui n'est autre qu'un état cérébral fonctionnellement défini par ses relations causales avec d'autres états cérébraux. Fodor peut donc dissocier les explications qui invoquent les attitudes propositionnelles du sens commun - la psychologie de Granny ${ }^{27}$ - des explications intentionnelles qui se contentent d'invoquer des ètats intentionnels ou représentationnels. Fodor souscrit généralement à la thèse réaliste scientifique selon laquelle les succès explicatifs et prédictifs d'une théorie scientifique sont autant d'arguments en faveur

26. Dans les théories de la covariation, un effet transmet une information sur sa cause. Mais informer n'est pas représenter. La représentation, non l'information, peut être fallacieuse. Pour Fodor, le contenu ou la représentation est une information qui obéit à la dépendance causale asymétrique. Le symbole mental « vache » dénote des vaches et non des chevaux même si certains chevaux la nuit occupent une extrémité d'une chaîne causale dont l'autre extrémité est occupée par le symbole « vache », car la relation nomique entre des chevaux et le symbole « vache » est asymétriquement dépendante par rapport à la relation nomique entre les vaches et le symbole « vache ». Cf. Psychosemantics, chapitre 4 et A Theory of Content, chapitre 3 .

27. Pour les exégètes de Fodor, Granny est le porte-parole du sens commun. Auntie est le porte-parole de ce que Fodor prend pour l'establishment physicaliste. 
d'une interprétation réaliste de ladite théorie. Du point de vue de son incorporation dans une théorie psychologique scientifique, l'adoption de l'interprétation réaliste des explications en termes d'attitudes propositionnelles - la psychologie du sens commun ou de Granny - (défendue au chapitre i de Psychosemantics) est plus conservatrice que le réalisme intentionnel. De ce dernier point de vue, « ce qui est intéressant dans le contenu intentionnel, c'est son aptitude à survivre aux variations de rôle fonctionnel $\gg^{28}$. Le réalisme des attitudes propositionnelles suppose que les généralisations causales de la psychologie scientifique mentionneront les concepts d'attitudes propositionnelles. Le réalisme intentionnel suppose que les généralisations causales de la psychologie scientifique mentionneront le contenu intentionnel. Le réalisme intentionnel, contrairement au réalisme des attitudes propositionnelles, est compatible avec l'idée que la psychologie scientifique réformera la psychologie de Granny.

\section{Comment concilier le rejet du holisme du contenu et la propriété de systématicité des pensées?}

Dans le cours de sa critique du holisme du contenu, Fodor s'adresse à lui-même deux objections parentes visant à réduire à l'absurde la séparation du contenu intentionnel d'Avec le rôle fonctionnel. Première objection : «si le contenu d'une croyance est indépendant de son rôle fonctionnel, alors le fait de croire que $p$ est compatible avec le fait de croire pratiquement n'importe quoi d'autre; $y$ compris non $p{ }^{29}$. Au lieu de tenir ce conditionnel pour la prémisse d'un modus tollens dont la sous-conclusion consisterait à rejeter le divorce entre le contenu et le rôle fonctionnel pour conclure que le refus du holisme est suspect, de manière caractéristique, Fodor traite le conditionnel comme la prémisse d'un modus ponens et « embrasse » la conclusion que le fait de croire que $p$ est compatible avec le fait de croire n'importe quoi d'autre : «j'admets - en fait, j'approuve - la conclusion selon 
laquelle les gens peuvent croire des choses qui sont arbitrairement insensées $>^{30}$.

Deuxième objection : si le contenu est divorcé du rôle fonctionnel, pourquoi devrions-nous tirer quelque conséquence que ce soit de nos pensées? « Avoir la pensée que trois est un nombre premier pourrait constituer une vie mentale entiere $\rangle^{31}$. De manière non moins caractéristique que précédemment, Fodor convertit le modus tollens de son adversaire en modus ponens et embrasse la conséquence destinée par son adversaire à servir de prémisse pour rejeter le divorce entre le contenu et le rôle fonctionnel.

La réponse de Fodor à la seconde objection soulève une difficulté qui tient à ce que l'exemple d'une pensée qui pourrait constituer une vie mentale entière est mal choisi. Dans une note $^{32}$. Fodor met à profit sa distinction entre une sémantique fonctionnaliste du contenu (qu'il rejette) et la conception psychofonctionnaliste des états de croyance (qu'il accepte) pour distinguer entre le fait d'avoir la croyance que trois est un nombre premier et le fait d'avoir la pensée (entertain the thought) que trois est un nombre premier. Avoir une croyance, c'est être dans un état fonctionnel défini par des relations causales avec des entrées, des sorties et d'autres états mentaux. Donc, un organisme ne pourrait pas croire que trois est un nombre premier sans avoir d'autres croyances. Admettons qu'en général on puisse effectivement distinguer le fait qu'une créature ait une croyance et le fait qu'elle ait une pensée. Supposons que le fait, pour une créature, d'avoir une croyance avec un contenu déterminé s'analyse au moins comme le fait que cette créature ait un symbole mental ayant le contenu pour propriété sémantique et ayant à l'égard de ce contenu une attitude définie fonctionnellement par son rôle causal à l'égard des entrées, des sorties et d'autres états mentaux. Admettons que le fait que la créature ait une (simple) pensée (sans croyance) avec un contenu déterminé se réduise au fait que la créature ait un symbole mental avec ce contenu sans avoir à l'égard de ce contenu

\footnotetext{
3o. Ibid.

31. Ibid., p. 89.

32. Ibid., p. I6r.
} 
l'attitude de croyance définie fonctionnellement. Pour que la pensée puisse constituer une vie mentale entière, il faut que son contenu soit une propriété sémantique primitive d'un symbole mental dont l'occurrence (dans le langage de la pensée de la créature) ne dépende que d'interactions causales entre la créature et son environnement physique. Le symbole dont le contenu constitue la vie mentale entière de la créature ne peut avoir un contenu complexe formé par composition à partir d'autres symboles mentaux plus élémentaires. Faute de quoi la pensée considérée ne constituerait pas la vie mentale entière de la créature. L'exemple parait donc mal choisi, car si on peut admettre que certaines pensées perceptuelles primitives puissent être le contenu de symboles dont l'occurrence ne dépend que d'interactions causales entre un organisme et son environnement, on ne comprend pas comment une pensée ayant le contenu arithmétique que trois est un nombre premier pourrait être la propriété sémantique d'un symbole mental primitif dont l'occurrence ne dépend que de l'interaction causale entre la créature et son environnement physique.

La seconde difficulté que je veux examiner concerne la compatibilitê entre la propriété de systématicité que Fodor attribue aux pensées et sa répudiation du holisme du contenu. La systématicité est cette propriété des pensées en vertu de laquelle une créature capable de penser que Marie aime Jean doit aussi être capable de penser que Jean aime Marie (indépendamment de la valeur de vérité des pensées) ${ }^{33}$. On pourrait se demander comment la systématicité s'applique à la pensée que Jean marche. Peut-être la systématicité implique-t-elle qu'une créature capable de penser que Jean marche doive être capable de penser que Jean ne marche pas ou que quelqu'un marche. Appelons « corrélats systématiques » d'une pensée $P$ les pensées qu'une créature dont les pensées sont systématiques doit être capable de former à chaque fois qu'elle a la pensée que $P$. La question soulevée est de

33. La propriété de systématicité n'est pas sans évoquer le principe de généralité de Evans. Mais comme nous allons le voir sous peu, il existe une différence de statut entre les deux notions. 
savoir si on peut distinguer le holisme du contenu de la notion de corrélat systématique d'une pensée.

Fodor se sert de la systématicité des pensées pour faire valoir la supériorité explicative de l'hypothèse (empirique) du langage de la pensée sur le simple réalisme intentionnel ${ }^{34}$ : le réalisme intentionnel postule la réalité des contenus intentionnels sans pouvoir expliquer la systématicité ou expliquer pourquoi une créature ne pourrait pas penser un membre d'une paire (ou d'un n-uple) de penseees s'il ne pouvait pas penser l'autre; le langage de la pensée suppose que les contenus intentionnels ont une structure syntaxique (en constituants) de sorte que deux pensées, comme la pensée que Marie aime Jean et la pensée que Jean aime Marie, peuvent combiner dans des ordres syntaxiques différents les mêmes constituants. Attribuer la systématicité aux pensées, n'est-ce pas ipso facto supposer qu'une pensée ne peut pas constituer une vie mentale entière? N'est-ce pas supposer que les pensées viennent par paquets? N'est-ce pas rejeter la possibilité de créatures ayant des vies mentales qui, du point de vue de la nôtre, semblent avoir des trous - des vies mentales «pointillées » (punctate minds) ? En un mot, la systématicité des pensées n'est-elle pas le holisme du contenu sous un autre nom?

Pour répondre à ces questions, il faut procéder en deux temps. Premièrement, il y aurait, me semble-t-il, un conflit direct entre la théorie dénotationnelle des contenus de pensée (ou la répudiation du holisme du contenu) et la thèse de la systematicité des pensées si les deux points de vue s'appliquaient à une seule et mème chose. Mais il me semble que Fodor est en droit de faire valoir que la théorie dénotationnelle est une théorie des contenus primitifs lou que sa répudiation du holisme du contenu vaut pour les contenus primitifs), tandis que la systématicité est manifestement une propriété des pensées un tant soit peu complexes - formées par composition de pensées primitives. Deuxièmement, l'attribution de la systématicité à la pensée humaine n'est ni une vérité nécessaire ni une vérité conceptuelle (découvrable a priori) ; c'est une vérité contingente que seule la recherche empirique

34. Cf. Psychosemantics. Appendix, « Why There Still Has to Be a Language of Thought ». 
permettra de corroborer ou d'infirmer. Si la systématicité est une propriété contingente des pensées humaines complexes (que la pensée humaine pourrait ne pas posséder), alors elle n'interdit nullement la possibilité conceptudle qu'une seule pensée (primitive) constitue la vie mentale entière d'une créature donnée. Comme le dit Fodor,

1... la systématicité est au moins quelques fois un trait contingent de la pensée : $\mid . .$. il y a au moins quelques cas dans lesquels il est possible pour une créature de penser l'une mais non l'autre de deux propositions unies par des relations de contenu $|. .$.$| Je veux toutefois rester$ neutre sur la question de savoir si la systematicité est toujours un trait contingent de la pensée l... ${ }^{35}$.

La contingence de la systématicité de la pensée est compatible avec la possibilité qu'une pensée (primitive) constitue la vie mentale entière d'une créature. Elle distingue d'une part la systématicité du principe de généralité qu'Evans tenait pour un principe constitutif ou transcendantal de la pensée. A la différence d'autre part d'un principe a priori de rationalité (comme le principe de charité mentionné par Quine, Davidson et Dennett ou le principe d'humanité mentionné par Grandy), la systématicité n'est pas un principe d'attribution de croyances. Quine, Davidson et Dennett font valoir que faute de créditer à des créatures la rationalité, un attributeur se priverait des moyens de recommaître une créature ayant des croyances. Ies normes de rationalité qui gouvernent nos attributions de croyances ne sont, selon eux, ni optionnelles ni réfutables. La distinction entre la systematicité et la rationalité est parallèle à la distinction fodorienne entre les contenus intentionnels complexes (non primitifs) et les états de croyance (ou les attitudes propositionnelles):

Il n'est pas plausible de supposer que les « principes de charité » contraignent les attributions intentionnelles per se, mème s'il est plausible de supposer qu'ils contraignent les attributions d'états de croyance. En particulier, on ne peut pas concevablement exiger que les objets propositionnels de toutes les attitudes attribuées à une personne à un instant soient mutuellement cohérents. Il n'y a rien à reprocher à quelqu'un qui espère que $P$ tout en redoutant que non $P$; et croire que $P$ tout en soulıaitant que non $P$ définit pratiquement la condition humaine. $S i$ la rationalité exerce des contraintes sur les attributions d'attitudes propositionnelles, elles s'appliquent aux 
relations entre les attitudes, non aux relations entre leurs objets propositionnels ${ }^{36}$.

Pour faire s'évanouir la distinction entre la systématicité des pensées complexes et un principe de rationalité (comme le principe de charité) supposé gouverné nos pratiques d'attribution, il faut nier deux distinctions fodoriennes : la distinction entre contenu et état de croyance et la distinction entre état de croyance et attribution de croyance.

Récapitulons : dans le cadre fodorien, nous distinguons entre des contenus intentionnels (ou des pensées) primitifs et des contenus intentionnels complexes. La théorie dénotationnelle proprement dite s'applique aux premiers ; la systématicité aux seconds. Nous distinguons conjointement entre les états de croyance (ou les attitudes propositionnelles) et les contenus intentionnels (ou les objets propositionnels) de ces états (ou attitudes). Le psychofonctionnalisme s'applique aux premiers et le fonctionnalisme sémantique ne s'applique pas aux seconds ${ }^{37}$. Nous distinguons enfin entre les états de croyance (qui sont des états mentaux) et les attributions de croyance (qui sont des énoncés). La systématicité, non la rationalité, est une propriété des pensées (ou contenus) complexes. La rationalité est une norme qui guide les attributions de croyances ou d'attitudes propositionnelles en général.

\section{4. Ítats de crovance et attributions de crovance}

Nous sommes maintenant en mesure d'aborder la théorie fodorienne d'un exercice dans lequel excelle la psychologie du sens commun : les attributions de croyance. Selon la théorie de la référence directe, l'énoncé d'une phrase contenant un terme singulier exprime toujours une proposition « russellienne singulière », y compris dans les contextes de croyance. Autrement dit, la contribution du terme singulier à la proposition exprimée est toujours son référent - non un mode de présentation frégéen du référent. Considérons les énoncés des deux phrases (I) et (2) qui

36. SAIB, P. 174.

37. À l'exception de certains contenus « logiques » exprimables par des symboles dènués de référent. 
ne diffèrent l'une de l'autre qu'en ce que dans ( 2 ) « Romain Gary » est remplacé par le nom coréférentiel « Ajar »:

(I) Romain Gary était moustachu.

(2) Ajar était moustachu.

Selon la théorie de la référence directe, un énoncé de (I) exprime la même proposition qu'un énoncé de (2). Considérons maintenant un énoncé de (3) et un énoncé de (4):

(3) François croit que Romain Gary était moustachu.

(4) François croit que Ajar était moustachu.

( 1 ) est contenue dans (3) et (2) est contenue dans (4). Selon la théorie de la référence directe, les termes singuliers coréférentiels « Romain Gary » et « Ajar » contribuent à introduire leur unique référent aussi bien dans un énoncé de (3) et un énoncé de (4) que dans un énoncé de (I) et dans un énoncé de (2).

Dans « Sens et dénotation », Frege dirige l'argument suivant contre la théorie de la référence directe :

Lra] Si un énoncé de ( 1 ) et un énoncé de (2) exprimaient la même proposition, alors les énoncés d'attribution de croyance (3) et (4) (qui contiennent respectivement (I) et (2)) devraient toujours avoir la même valeur de vérité (pour une assignation donnée de référent à « Romain Gary 》 et « Ajar »).

[2al Or (3) et (4) peuvent avoir des valeurs de vérité différentes.

[3a] Donc : un énoncé de ( 1 ) et un énoncé de (2) n'expriment pas la même proposition.

Frege conclut que la contribution propositionnelle d'un terme singulier est son sens ou son mode de présentation du référent.

Théoricien de la référence directe, Salmon rejette explicitement la prémisse [2a]. En faveur de la prémisse [2a], le sens commun fait valoir que François peut donner des signes d'assentiment à la proposition exprimée par un énoncé de (I), énoncer lui-même la proposition en question et refuser son assentiment à la proposition exprimée par un énoncé de (2) ou énoncer la négation de la proposition exprimée par un énoncé de (2). Salmon soutient que si la proposition exprimée par un énoncé de (3) est vraie, alors automatiquement la proposition exprimée par un énoncé de (4) est vraie aussi. Selon lui, François a beau donner tous les 
signes imaginables qu'il ne tient pas pour vraie la proposition exprimée par un énoncé de (2) les deux énoncés d'attribution ont la même valeur de vérité.

La stratégie de Salmon est double. Elle consiste d'une part à maintenir que la forme logique de la proposition explicitement exprimée par une attribution de croyance comme (3) ou (4) est la suivante :

(Em) CR (François, p, m),

où « $C R$ » est un prédicat à trois places, « $\mathrm{p}$ » est la proposition singulière russellienne (exprimable par un énoncé de (I) ou de (2)) et « $\mathrm{m}$ » est le mode de présentation du référent du terme singulier (qui est aussi le mode de présentation de la proposition singulière elle-même).

Elle consiste d'autre part à soutenir que les propositions implicitement communiquées par un énoncé de (3) et par un énoncé de (4) contiennent une référence à deux modes de présentation distincts de Romain Gary ${ }^{38}$. Un énoncé de (3) et un énoncé de (4) expriment explicitement la même proposition vraie (qui contient une quantification existentielle sur les modes de présentation de Romain Gary). Mais de surcroît un énoncé de (3) communique implicitement la proposition vraie selon laquelle François reconnaît la proposition singulière sous le mode de présentation de Romain Gary associé à « Romain Gary », et un énoncé de (4) communique implicitement la proposition fausse selon laquelle François reconnaît la même proposition singulière sous le mode de présentation de Romain Gary associé à « Ajar ».

La faiblesse principale de la conception des attributions de croyance de Salmon tient au manque de justification de son révisionnisme: si Salmon a raison, alors l'usage ordinaire erre en supposant qu'un énoncé de (3) peut exprimer une proposition vraie et un énoncé de (4) une proposition fausse.

Dans SAIB, Fodor considère la version de l'argument de Frege contre la théorie de la référence directe (ou théorie dénotationnelle du contenu) recourant à la non-substituabilité d'expressions coréférentielles :

38. Salmon semble supposer que le mécanisme d'engendrement du contenu implicitement communiqué est un mécanisme à la Grice. 
[Ib] Le contenu exprimé par une expression complexe est une fonction du contenu de ses constituants.

[2bl Le contenu d'une expression est sa dénotation.

[3b] Deux expressions ayant le même contenu peuvent toujours être substituées l'une à l'autre dans une expression plus complexe sans altérer le contenu (la dénotation) de l'expression plus complexe ou la valeur de vérité de cette expression lorsque celle-ci est une phrase.

[4b] Comme le montrent les précédents exemples d'attribution (3) et (4), deux expressions ayant la même dénotation ne sont pas toujours substituables l'une à l'autre dans une phrase sans en altérer la valeur de vérité.

[5b] Frege conclut qu'il convient de rejeter [zb] et que le contenu d'une expression ne se réduit pas à sa dénotation.

Le but de Fodor est de défendre [ab] contre l'argument frégéen invoquant la non-substituabilité. Autrement dit, le but de Fodor est de montrer que la non-substituabilité d'expressions coréférentielles dans des contextes de croyance est compatible avec le fait que ces expressions expriment un seul et même contenu. Comme le contexte dans lequel s'effectue la substitution d'expressions coréférentielles est un contexte de croyance, la défense de la théorie dénotationnelle dépend d'une théorie des attributions de croyance. Avant de présenter sa théorie des attributions de croyance, Fodor raffine la théorie de l'individuation des états de croyance présentée dans Psychosemantics. Croire est traité comme une relation à quatre places qui prend pour arguments, outre (a) une personne, (b) une proposition (ou un contenu), (c) un véhicule (ou symbole mental) et (d) un rôle fonctionnel.

Le contenu propositionnel n'est autre que le contenu large. Le véhicule ou symbole mental correspond à ce qui est traditionnellement tenu pour un mode de présentation. Étant un symbole, le véhicule a des propriétés syntaxiques et des propriétés sémantiques. Deux véhicules concrets particuliers (tokens) appartiennent à des types distincts s'ils sont syntaxiquement différents ou s'ils servent à exprimer des contenus propositionnels différents. Deux véhicules concrets particuliers appartenant à des types distincts peuvent - comme les symboles des langues publiques exprimer une seule et même proposition. L'innovation principale 
de Fodor réside dans l'insertion du rôle fonctionnel comme paramètre de l'état de croyance. Le rôle fonctionnel (du véhicule) est son rôle inférentiel dans un système de croyances : « son rôle causal dans certains processus mentaux ${ }^{39}$. Deux véhicules concrets particuliers appartenant à un seul et même type auront des rôles fonctionnels distincts s'ils appartiennent à des systèmes de croyances différents. Réciproquement ${ }^{40}$, deux véhicules concrets particuliers appartenant à des types distincts layant des propriétés syntaxiques et sémantiques différentes) peuvent avoir un seul et même rôle fonctionnel, c'est-à-dire contribuer dans deux systèmes de croyances distincts à engendrer une seule et même croyance.

En tenant l'état de croyance pour une relation à quatre places. Fodor peut maintenir qu'une personne peut être dans deux états de croyance distincts à l'égard d'un seul et même contenu propositionnel. Il lui suffit pour cela d'attribuer la différence d'état de croyance à une différence entre les véhicules ou entre les rôles fonctionnels. La conception fodorienne n'a pas les implications révisionnistes (par rapport à l'usage ordinaire) de la théorie de Salmon que j'ai mises en lumière précédemment.

Du point de vue fodorien, on peut distinguer des attributions plus ou moins spécifiques selon qu'elles aspirent ou non à caractériser, outre le contenu, le véhicule. Une attribution qui aspire à spécifier le véhicule est plus spécifique qu'une attribution qui se contente de caractériser le contenu. Les normes qui, selon Fodor, guident un attributeur dans la spécification du véhicule sont moins contraignantes que celles qui président à la spécification du contenu :

En ce qui concerne la spécification de l'objet propositionnel de la croyance attribuée l...l, la formule enchâssée doit exprimer la proposition attribuée par le prédicat «croit » I... La spécification du véhicule dépend de facteurs pragmatiques glissants islipperyl. Ce qui est requis est un certain degré d'isomorphie avec le véhicule - degré d'isomorphie approprié pour une tâche déterminée. On ne peut pas dire indépendamment d'une tâche particulière quel degré d'isomorphie est suffisant ${ }^{4}$.

39. $S A I B$, p. 168 .

40. Ibid., note 8, p. 176 .

4I. Ibid., P. I7I. 
On peut représenter l'attribution la moins spécifique (qui n'aspire qu'à caractériser le contenu) au moyen d'une formule du type [AIl :

IAIl (Ex)(Ey) [(x est un véhicule \& y est un rôle) \& (CR (François, $P, x, y))$,

où « $\mathrm{P} \gg$ fait référence au contenu exprimé par un énoncé de (I) «Romain Gary était moustachu ». Une telle formule, qui fait référence au contenu propositionnel, contient une généralisation existentielle sur les véhicules et sur les rôles fonctionnels non spécifiés. Une telle formule s'apparente à ce qu'on nomme traditionnellement une attribution de re ou représente une attribution de contenu dit large. On peut représenter l'attribution plus spécifique (qui aspire à caractériser, outre le contenu, le véhicule) par [A2] :

[A2] (Ey) l(y est un rôle) E (CR (François, P, r, y))], où « $\mathrm{r} »$ fait référence au véhicule (ou au symbole mental) dans la tête de François « isomorphe » à la phrase française (I) « Romain Gary était moustachu ». Une telle formule, qui fait référence au contenu et au symbole mental, ne généralise plus que sur les rôles fonctionnels. Elle s'apparente à ce qu'on nomme traditionnellement une attribution de dicto et livre sur l'état de croyance plus d'information que le seul contenu large.

Supposons qu'on interprète un énoncé de (3) ou de (4) comme une attribution aussi peu spécifique que possible n'aspirant qu'à spécifier le contenu (large) de la croyance de François, sur le modèle de la double quantification existentielle contenue dans la formule [AI]. Dans cette interprétation, (3) et (4) ne contiennent aucune expression faisant référence au véhicule. Les expressions référentielles ont leurs références ordinaires : elles sont mutuellement substituables dans (3) et (4), et on peut tenir conjointement un énoncé de (3) et un énoncé de (4) pour vrais. Interprétons maintenant un énoncé de (3) ou de (4) comme une attribution plus spécifique aspirant à caractériser, outre le contenu, le véhicule. Dans ce cas, nous pourrions représenter respectivement (3) et (4) sur le modèle de la simple quantification existentielle contenue dans la formule $\mid A 2]$ :

[3'] (Ey) I(y est un rôle) \& (CR (François, P, r, y))], 
où « $\mathrm{r} \gg$ fait référence au véhicule dans la tête de François « isomorphe » à la phrase française (I) « Romain Gary était moustachu ».

[4'] (Ey) [(y est un rôle) E (CR (François, P, a, y))], où « a » fait référence au véhicule dans la tête de François « isomorphe » à la phrase française (2) « Ajar était moustachu ».

Dans cette interprétation, en dépit du fait que « Romain Gary » et « Ajar » sont coréférentiels lou que (i) et (2) ont la même valeur de vérité), $\left|3^{\prime}\right|$ serait une formule vraie et $\left[4^{\prime}\right]$ une formule fausse.

Il en résulte que la théorie fodorienne n'est pas exposée à l'objection de révisionnisme insuffisamment justifié que j'ai adressée à la théorie de Salmon. Toutefois, cette force est peutêtre aussi une faiblesse du point de vue de la perspective globale de Fodor sur la psychologie scientifique. En autorisant en effet deux représentations d'attributions spécifiques (aspirant à caractériser le véhicule, et non seulement le contenu), représentées par les formules [3'] et [4'], à avoir des valeurs de vérité différentes, Fodor concède que la sémantique des attributions plus spécifiques de croyance n'obéit pas strictement au principe de compositionnalité : le contenu de l'attribution - sa valeur de vérité - n'est pas une stricte fonction du contenu (de la dénotation) de ses constituants. Elle dépend donc d'un aspect non sémantique d'un ou plusieurs de ses constituants - non sémantique au sens fodorien dans lequel la totalité du contenu d'un état de croyance réside dans la proposition, indépendamment des propriétés syntaxiques du véhicule et du rôle fonctionnel. On peut attribuer la différence entre la valeur de vérité de [3'] et la valeur de vérité de [4'] à la différence entre les propriétés syntaxiques du symbole mental (ou du véhicule) dans la tête de François qui est isomorphe respectivement à « Romain Gary » et à « Ajar ». Du point de vue de la perspective globale de Fodor sur la psychologie scientifique, on peut admettre que le succès explicatif d'une attribution de croyance dépende conjointement de la capacité de l'attribution à faire référence au contenu intentionnel et aux propriétés syntaxiques du véhicule dont le contenu est la propriété sémantique. Conformément à la condition de formalité, en effet, un symbole mental tient son rôle causal de ses propriétés syntaxiques. 
Mais considérons la généralisation ceteris paribus du sens commun suivante: «Si une personne $X$ forme perceptuellement la croyance qu'un taureau de $400 \mathrm{~kg}$ est sur le point de la charger. toutes choses égales, elle formera le désir de se mettre à distance respectable de la trajectoire du taureau. » L'antécédent d'une telle généralisation contient une attribution de croyance qui, du point de vue fodorien, s'analyse comme une relation entre une personne, une proposition $P$ (qu'un taureau de $400 \mathrm{kgs}$ est sur le point de charger $\mathrm{X}$ ), un véhicule et un rôle fonctionnel. Le conséquent contient une attribution de désir qui s'analyse comme une relation entre une personne, une proposition $Q$ (que $X$ se mette à distance respectable de la trajectoire du taureau), un véhicule et un rôle fonctionnel.

À la différence des attributions spécifiques [3'] et [4'], dont la valeur de vérité (et l'efficacité explicative) dépend - outre de la référence au contenu - des propriétés syntaxiques du véhicule, le succès explicatif de la généralisation peut dépendre du rôle fonctionnel de la croyance attribuée. La généralisation expliquera avec succès le comportement d'une personne qui préfère maximiser la distance qui la sépare d'un taureau de $400 \mathrm{~kg}$ qui charge au galop dans sa direction. Mais un individu peut d'une part croire qu'une personne est sur la trajectoire d'un taureau de $400 \mathrm{~kg}$ qui charge dans sa direction sans croire qu'il est lui-même cette personne. Imaginez un système compliqué de miroirs grâce auxquels vous pourriez voir une personne chargée par un taureau sans réaliser que vous êtes vous-même cette personne. Une personne peut d'autre part croire qu'elle est sur la trajectoire d'un taureau de 400 $\mathrm{kg}$ lancé au plein galop et préférer maximiser la proximité avec le taureau - parce que, par exemple, cette personne est un torero ou parce qu'elle a un système de croyances dans lequel est contenue celle selon laquelle l'âme d'une personne piétinée par un taureau ira droit au paradis, et que, toutes choses égales, cette personne désire que son âme aille droit au paradis. Une personne dont le système de croyances inclut la croyance que l'âme d'une personne piétinée par un taureau ira droit au paradis et dont le système de préférences inclut le désir que son âme aille droit au paradis peut avoir la croyance qu'un taureau de $400 \mathrm{~kg}$ charge au galop dans sa direction. Elle aura alors, selon Fodor, une croyance ayant le mème contenu intentionnel que la croyance d'une autre 
personne qui ne croit pas par ailleurs que l'âme d'une personne piétinée par un taureau ira droit au paradis. Le véhicule grâce auquel la personne $X$ (qui croit que l'âme d'une personne piétinée par un taureau ira droit au paradis) croit qu'un taureau de $400 \mathrm{~kg}$ charge au galop dans sa direction peut être un symbole mental du même type que le véhicule grâce auquel une personne $Y$ (qui ne croit pas que l'âme d'une personne piétinée par un taureau ira droit au paradis) a la croyance qu'un taureau de $400 \mathrm{~kg}$ charge au galop dans sa direction. La différence entre l'état de croyance d'une personne $X$ (qui croit que l'âme d'une personne piétinée par un taureau ira au paradis) et l'état de croyance d'une personne $Y$ (qui ne croit pas que l'âme d'une personne piétinée par un taureau ira droit au paradis) lorsqu'elles croient toutes les deux qu'un taureau de $400 \mathrm{~kg}$ charge au galop dans leur direction provient de l'insertion de deux véhicules du même type dans des systèmes de croyances distincts.

La différence de comportement entre une personne $X$ qui a la croyance selon laquelle l'âme d'une personne piétinée par un taureau ira droit au paradis et une personne $Y$ qui n'a pas cette croyance n'est pas un mystère. Cette différence de croyance explique leur différence de comportement. Mais la théorie fodorienne des attributions de croyance n'en est pas moins, me semble-t-il, confrontée à la question suivante : étant admis que les deux personnes ont des systèmes de croyances distincts, lorsqu'elles forment toutes les deux la croyance qu'un taureau de $400 \mathrm{~kg}$ charge au galop dans leur direction respective, ont-elles ou n'ont-elles pas la même croyance? Étant donné que, pour Fodor, un état de croyance est une relation entre une personne, un contenu, un véhicule et un rôle fonctionnel, Fodor doit répondre de la manière suivante : d'une part ces deux personnes sont dans des états de croyance distincts puisque leurs véhicules respectifs sont insérés dans des systèmes de croyances différents et ont des rôles fonctionnels distincts ; d'autre part ces états de croyance distincts partagent le même contenu ${ }^{42}$.

42. Ce paragraphe a été provoqué par une critique de Claude Panaccio après mon exposé à l'UQAM. 
À ce stade, Fodor me semble confronté au dilemme suivant : soit Fodor place le rôle fonctionnel d'une croyance du côté de son efficacité causale; soit il le place du côté du contenu. Première branche de l'alternative : Fodor suppose que le potentiel d'interaction inférentielle d'une croyance (la croyance qu'un taureau de $400 \mathrm{~kg}$ charge au galop dans la direction de la personne ayant la croyance) fait partie du rôle causal de la représentation mentale. Auquel cas, il doit concéder que le rôle causal d'une représentation mentale (un véhicule) ne se réduit pas, contrairement au vœu de « Methodological Solipsism », à ses propriétés purement syntaxiques. Peut-être pourrait-il, dans cette hypothèse, distinguer un rôle causal étroit (dévolu aux seules propriétés syntaxiques du symbole) et un rôle causal large (dévolu au rôle fonctionnel du symbole). Deuxième branche de l'alternative: Fodor nie que le rôle fonctionnel ait une efficacité causale et il tient le rôle fonctionnel pour un ingrédient du contenu du symbole mental. Mais dans ce cas, il doit renoncer à maintenir une théorie purement dénotationnelle du contenu et il doit nuancer sa répudiation du holisme du contenu.

Centre de Recherche en Epistémologie Appliquée Ecole Polytechnique, Paris 